Pizzo MD, Pediatric Branch, National Cancer Institute, Bldg 10, Rm 13N240, 900 Rockville Pike, Bethesda, MD 20892).

COMMENT. CNS involvement in children with HIV infection is common and is frequently manifested with cognitive dysfunction or regression of developmental milestones. Calcifications in the basal ganglia and frontal lobes, white matter changes, and cerebral atrophy are the usual MRI findings. Cerebrovascular disease is an uncommon but potentially hazardous complication. ( See Ped Neur Briefs Aug 1991; $\underline{5}$ : 57-58, for further discussion of CNS involvement in HIV infection).

A recent neuropsychologic study of HIV-infected children with hemophilia at the University of North Carolina, Chapel Hill, suggested an overall trend toward lower motor, attentional, memory, and sensoryperceptual functioning. When the results were contrasted to those in a control group of children who also had hemophilia, no significant differences were apparent. The early, subtle psychological deficits could not be attributed solely to CNS effects of HIV infection. (Whitt JK et al. Neuropsychologic functioning of human immunodeficiency virus-infected children with hemophilia. I Pediatr Jan 1993; 122: 52-59). (Reprints: JK Whitt MD, Dept of Psychiatry, Campus Box No 7160, University of North Carolina, Chapel Hill, NC 27599).

\title{
MULTIPLE ARTERIOVENOUS SHUNTS
}

Symptoms, management, and outcome of 13 cases of multiple cerebral arteriovenous malformations (MAVMs) are reported from the Centre Hospitalier de Bicetre, France. The age ranged from 6 months to 15 years. The incidence of MAVM among the pediatric population with brain AVMs was $16.9 \%$. Congenital and acquired types were distinguished. Hemorrhage was the presenting symptom in 31\%. Spontaneous regression occurred in $15 \%$. Angiogenesis, or sprouting around a true AVM following hemorrhage, may account for some acquired MAVMs. Embolization was the most successful mode of treatment, but anatomical cure was rarely obtained. (Iizuka Y et al. Multiple cerebral arteriovenous shunts in children: report of 13 cases. Child's Nerv Syst Dec 1992; 8: 437-444). (Correspondence: P Lasjaunias MD, Service de Neuroradiologie, Centre Hospitalier de Bicetre, 78, Rue du General Leclerc, F-94275 Le Kremlin Bicetre Cedex, France).

COMMENT. The rate of serious morbidity following a hemorrhage from an AVM is about $30 \%$ and the mortality is about $10 \%$. AVMs in children are more apt to bleed than those in adults. Total excision seems the treatment of choice when feasible. (See Progress in Pediatric Neurology, Ed Millichap JG, Chicago, PNB Publishers, 1991). With multiple AVMs in the present study, embolization was preferred. 